J. Austral. Math. Soc. (Series A) 29 (1980), 17-28

\title{
THE ROOT SYSTEM OF PRIMES OF A HAHN GROUP
}

\author{
MARLOW ANDERSON and OTIS KENNY
}

(Received 18 April 1979)

Communicated by J. B. Miller

\begin{abstract}
Let $\Delta$ be a root system and let $V$ be the Hahn group of real-valued functions on $\Delta$. Then $\Delta$ can be order-embedded into $P(\Delta)$, the root system of prime $l$-ideals of $V$. In this note we identify $P(\Delta)$ in terms of $\Delta$ without explicit reference to $V$, up to the convex subgroup structure of the additive groups of real closed $\eta_{1}$-fields. In particular, we characterize the minimal prime $l$-ideals of $V$ in terms of $\Delta$ by an ultrafilter construction which generalizes the well-known method when $\Delta$ is trivially ordered.
\end{abstract}

1980 Mathematics subject classification (Amer. Math. Soc.): 06 F 20.

\section{Introduction}

Throughout this introduction let $G$ be an abelian $l$-group. Let $\mathscr{C}(G)$ denote the set of all convex $l$-subgroups of $G$ (or l-ideals, since they are normal). If $G$ is an $l$-subgroup of an l-group $H$, and the map $C \rightarrow C \cap G$ is a lattice isomorphism of $\mathscr{C}(H)$ onto $\mathscr{C}(G)$, then $H$ is an a-extension of $G$. Those elements $P$ of $\mathscr{C}(G)$ for which $G / P$ is totally ordered are called prime; equivalently, the set of elements of $\mathscr{C}(G)$ larger than $P$ is a chain. Thus, the set of primes forms a root system, that is, a partially ordered set in which no two incomparable elements have a lower bound. Each prime exceeds at least one minimal prime; a prime $P$ is minimal if and only if for each $g \in P^{+}$there exists $h \notin P^{+}$such that $h \wedge g=0$. If the intersection $P^{*}$ of all elements of $\mathscr{C}(G)$ larger than a prime $P$ covers $P$, then $P$ is called a value; it is maximal with respect to not containing each element $g \in P^{*} \backslash P$. The root system of all values of $G$ is denoted by $\Gamma(G)$. If $\Delta$ is any root system, then

$$
V=V(\Delta, \mathbf{R})=\{f: \Delta \rightarrow \mathbf{R}: \text { the support of } f \text { has } A C C\}
$$


is an abelian l-group, called a Hahn group. Each abelian l-group $G$ may be $l$ embedded into $V(\Gamma(G), \mathbf{R})$ (Conrad, Harvey and Holland (1963)). For further information about $l$-groups, the reader may consult Conrad (1970), or Bigard, Keimel and Wolfenstein (1977).

If $\Delta$ is a trivially ordered set, then it is well known that the set of minimal primes of $V(\Delta, \mathbf{R})$ are in a one-to-one correspondence with the ultrafilters on $\Delta$ (see Conrad and McAlister (1969) and Gillman and Jerison (1960)). In the next section we generalize this to the case where $\Delta$ is any root system. In the third section, we identify the set $P(\Delta)$ of the prime l-ideals of $V$ in two steps. First, we identify $S(\Delta)=P(\Delta) / \approx$, where $P \approx Q$ if they contain the same set of minimal primes. (For a discussion of this equivalence relation in a more general context, see Conrad (1978).) Then, each $\approx$-equivalence class is described in terms of the convex subgroup structure of the additive groups of certain real closed $\eta_{1}$-fields.

\section{The minimal prime $l$-ideals of $V$}

Throughout $\Delta$ will be a fixed root system and $V=V(\Delta, \mathbf{R})$. Let $\mathfrak{A}$ be the set of all maximal trivially ordered subsets of $\Delta$. We partially order $\mathfrak{A}$ by declaring $A \leqslant B$ if $\delta \in A$ implies that there exists $\gamma \in B$ with $\delta \leqslant \gamma$. This is a lattice order with

and

$$
A \vee B=\{\text { maximal elements of } A \cup B\}
$$

$$
A \wedge B=\{\text { minimal elements of } A \cup B\} .
$$

We will occasionally abuse this notation by speaking of $A \vee B$ where at most one of $A$ and $B$ is trivially ordered but not maximal. If $A, B \in \mathfrak{U}$ and $X \subseteq A \cap(B \wedge A)$, let

$$
B^{\leftarrow}(X)=\{\delta \in B: \text { there exists } \gamma \in X \text { with } \gamma \leqslant \delta\} .
$$

Lemma 2.1. Let $A, B \in \mathfrak{A}$ with $A \leqslant B$ and let $\mathscr{U}$ be an ultrafilter on $A$. Let $B^{\leftarrow}(\mathscr{U})=\left\{B^{\leftarrow}(X): X \in \mathscr{U}\right\}$. Then $B^{\leftarrow}(\mathscr{U})$ is an ultrafilter on $B$.

Proof. Clearly $B^{\leftarrow}(X) \neq \varnothing$ for each $X \in \mathscr{U}$. Suppose $X, Y \in \mathscr{U}$ and let

and

$$
U=\bigcup\left\{W \subseteq A: B^{\leftarrow}(W)=B^{\leftarrow}(X)\right\}
$$

$$
Z=\bigcup\left\{W \subseteq A: B^{\leftarrow}(W)=B^{\leftarrow}(Y)\right\}
$$

Then, $U, Z \in \mathscr{U}, B^{\leftarrow}(U)=B^{\leftarrow}(X), B^{\leftarrow}(Z)=B^{\leftarrow}(Y)$, and $B^{\leftarrow}(U) \cap B^{\leftarrow}(Z)=B^{\leftarrow}(U \cap Z)$. Since $U \cap Z \in \mathscr{U}, B^{\leftarrow}(X) \cap B^{\leftarrow}(Y)=B^{\leftarrow}(U \cap Z)$, which is an element of $B^{\leftarrow}(\mathscr{U})$. 
Therefore, $B^{\leftarrow}(\mathscr{U})$ has the finite intersection property. Similarly, if $X \subseteq B$, then $X \in B^{\leftarrow}(\mathscr{U})$ or $B \backslash X \in B^{\leftarrow}(\mathscr{U})$. Therefore $B^{\leftarrow}(\mathscr{U})$ is an ultrafilter.

If $A(\mathscr{U})$ and $B(\mathscr{U})$ are ultrafilters on $A, B \in \mathfrak{U}$ respectively, then $A(\mathscr{U})$ and $B(\mathscr{U})$ are said to be compatible if

$$
(A \vee B)^{\leftarrow}(A(\mathscr{U}))=(A \vee B)^{\leftarrow}(B(\mathscr{U}))
$$

If for each $A \in \mathfrak{A}, A(\mathscr{U})$ is an ultrafilter on $A$, and for each $A, B \in \mathfrak{H}, A(\mathscr{U})$ and $B(\mathscr{U})$ are compatible, then $\{A(\mathscr{U}): A \in \mathfrak{U}\}$ is called a compatible system of ultrafilters on $\mathfrak{A}$.

For each $v \in V$, let $S(v)=\{\alpha \in \Delta: v(\alpha) \neq 0\}$ and let

$$
M(v)=\{\text { maximal elements of } S(v)\} .
$$

Since $v \in V, S(v)$ satisfies the ascending chain condition and so $\delta \in S(v)$ implies that there exists $\alpha \in M(v)$ such that $\alpha \geqslant \delta$. Clearly $M(v)$ is a trivially ordered set.

THEOREM 2.2. There is a one-to-one correspondence between minimal prime l-ideals of $V$ and compatible systems of ultrafilters on $\mathfrak{A}$ given as follows:

Let $P$ be a minimal prime l-ideal of $V$. For each $A \in \mathfrak{A}$, let $A(\mathscr{U})=\{A \backslash M(v): v \in P\}$. Then $\mathscr{C}_{P}=\{A(\mathscr{U}): A \in \mathfrak{A}\}$ is a compatible system of ultrafilters on $A$.

Let $\mathscr{C}=\{A(\mathscr{U}): A \in \mathfrak{Q}\}$ be a compatible system of ultrafilters on $\mathfrak{A}$ and let

$$
P_{\mathscr{C}}=\{v \in V: A \backslash M(v) \in A(\mathscr{U}), \text { for all } A \in \mathfrak{A}\} .
$$

Then $P_{\mathscr{C}}$ is a minimal prime l-ideal of $V$.

Proof. Let $P$ be a minimal prime; we first show that each element $A(\mathscr{U})$ of $\mathscr{C}_{P}$ is an ultrafilter on $A$. Suppose $\emptyset \in A(\mathscr{U})$. Then there exists $v \in P^{+}$so that $M(v) \supseteq A$. Since $A$ is a maximal trivially ordered set, $M(v)=A$. But then $w \wedge v=0$ implies that $w=0$, which is impossible since $v$ is an element of the minimal prime $P$. Therefore, each element of $A(\mathscr{U})$ is non-empty. Now let $X, Y \in A(\mathscr{U})$ and choose $u, v \in P^{+}$such that $A \backslash M(u)=X$ and $A \backslash M(v)=Y$. Let $s=\chi(A \backslash X)$ and $t=\chi(A \backslash Y)$ where $\chi(T)$ is the characteristic function on $T$. Then $x=s \wedge u$ and $y=t \wedge v$ are both elements of $P$. Moreover, $M(x)=A \cap M(u)$ and $M(y)=A \cap M(v)$ and so $M(x \vee y)=M(x) \cup M(y)$. Therefore,

$$
\begin{aligned}
X \cap Y & =(A \backslash M(u)) \cap(A \backslash M(v))=(A \backslash M(x)) \cap(A \backslash M(y)) \\
& =A \backslash(M(x) \cup M(y))=A \backslash M(x \vee y) .
\end{aligned}
$$

Since $x \vee y \in P, X \cap Y \in A(\mathscr{U})$ and so $A(\mathscr{U})$ has the finite intersection property. Finally, let $X \subseteq A$, and suppose that $u=\chi(X)$ and $v=\chi(A \backslash X)$. Then $u \wedge v=0$; so 
$u \in P$ or $v \in P$. Therefore $A \backslash X \in A(\mathscr{U})$ or $X \in A(\mathscr{U})$ and so $A(\mathscr{U})$ is an ultrafilter on $A$.

Next, we show that $\mathscr{C}_{P}$ is a compatible system of ultrafilters on $\mathfrak{A}$. Let $A, B \in \mathfrak{U}$ with $A \leqslant B$. We need to show that $B(\mathscr{U})=B^{\leftarrow}(A(\mathscr{U})$ ). Suppose (by way of contradiction) that there is $B \backslash X \in B(\mathscr{U})$ with $X \in B^{\leftarrow}(A(\mathscr{U}))$. Let $u=\chi(X)$ and $v=\chi(B \backslash X)$. Then $u \wedge v=0$ and so we may assume that $u \in P$. Let

$$
U=\bigcup\left\{W \in A(\mathscr{U}): B^{\leftarrow}(W)=X\right\}
$$

Then $U \in A(\mathscr{U})$ and if $W=\chi(U)$, then $0<w \leqslant u$ and so $w \in P$. Since $U \in A(\mathscr{U})$, by the argument above there exists $x \in P^{+}$so that $A \backslash U=M(x)$. But then $x \vee w \in P$ and $M(x \vee w)=A$, which is impossible, since $P$ is a minimal prime. Therefore, $\mathscr{C}_{P}$ is a compatible system of ultrafilters on $\mathfrak{A}$.

Now, let $\mathscr{C}=\{A(\mathscr{U}): A \in \mathfrak{A}\}$ be a compatible system of ultrafilters on $\mathfrak{A}$; we shall show that $\boldsymbol{P}_{\mathscr{C}}$ is a minimal prime. Let

$$
Q=\{v \in V: \text { for all } A \in \mathfrak{X} \text { with } M(v) \subseteq A, A \backslash M(v) \in A(\mathfrak{U})\}
$$

We will simplify the computations which follow by first showing that $P=Q$. Clearly $P \subseteq Q$. Suppose by way of contradiction that $v \in Q^{+} \mid P$. Then there is a $B \in \mathfrak{A}$ with $B \backslash M(v) \notin B(\mathscr{U})$. Since $B(\mathscr{U})$ is an ultrafilter, $B \cap M(v) \in B(\mathscr{U})$. Let $A \in \mathfrak{A}$ be such that $M(v) \subseteq A$. Therefore $B \cap M(v) \subseteq A$ and so $B \cap M(v) \in(A \vee B)(\mathscr{U})$. Since $v \in Q, X=A \backslash M(v) \in A(\mathscr{U})$; so $(A \vee B)^{\leftarrow}(X) \in(A \vee B)(\mathscr{U})$. However,

$$
(B \cap M(v)) \cap\left((A \vee B)^{\leftarrow}(X)\right)=\varnothing,
$$

which is impossible since $(A \vee B)(\mathscr{U})$ is an ultrafilter. Therefore, $Q=P$. Since $\mathscr{C}$ is a compatible system of ultrafilters,

$$
P=\{v \in V: \text { there exists } A \in \mathfrak{U} \text { with } A \supseteq M(v) \text { and } A \backslash M(v) \in A(\mathscr{U})\}
$$

With this simplification of the definition of $P$, we will proceed with the proof.

$P$ is a subgroup. Let $u, v \in P$ and let $x=u+v$. Let $A, B, C \in \mathscr{U}$ be such that $M(x) \subseteq A, M(u) \subseteq B$ and $M(v) \subseteq C$. By replacing $A$ by $A \wedge(B \vee C)$ we may assume that $A \leqslant B \vee C$. Since $u, v \in P,(B \vee C) \backslash M(u) \in(B \vee C)(\mathscr{U})$ and $(B \vee C) \backslash M(v) \in(B \vee C)(\mathscr{U})$. Therefore,

$$
(B \vee C) \backslash(M(u) \cup M(v)) \in(B \vee C)(\mathscr{U})
$$

Let $X=(B \vee C)^{\leftarrow}(M(x))$. Then $X \subseteq M(u) \cup M(v)$ and so $X \notin(B \vee C)(\mathscr{U})$. Therefore $M(x) \notin A(\mathscr{U})$ and thus $x \in P$. Since $M(x)=M(-x), x \in P$ implies that $-x \in P$. Therefore $P$ is a subgroup. 
$P$ is convex. Suppose $0<x<u$ and $u \in P$. Let $A, B \in \mathscr{U}$ be chosen so that $M(x) \subseteq A$ and $M(u) \subseteq B$. Without loss of generality, $A \leqslant B$. Since $B^{\leftarrow}(M(x)) \subseteq M(u)$, $B^{\leftarrow}(M(x)) \notin B(\mathscr{U})$ and thus $M(x) \notin A(\mathscr{U})$. Therefore $x \in P$.

$P$ is a minimal prime. Since $M(u)=M(|u|), u$ in $P$ implies that $|u| \in P$. Since $P$ is a convex subgroup, this means that $P$ is an $l$-subgroup. Let $u, v \in V$ be chosen so that $u \wedge v=0$. Pick $A \in \mathfrak{A}$ so that $M(u) \cup M(v) \subseteq A$. Since $M(u) \cap M(v)=\varnothing$, $A \backslash M(u)$ or $A \backslash M(v)$ is an element of $A(\mathscr{U})$. Thus $u$ or $v$ is in $P$ and so $P$ is prime. A similar argument will show that $v \in P^{+}$implies the existence of $u \notin P$ such that $u \wedge v=0$; thus $P$ is a minimal prime.

\section{The structure of $P(\Delta)$}

Let $P(\Delta)$ be the set of prime $l$-ideals of $V(\Delta, \mathbf{R})$, and $m(\Delta)$ the set of minimal primes of $V$. From Section 2, we know that $m(\Delta)$ is order-isomorphic to $\vec{m}(\Delta)$, the set of compatible systems of ultrafilters on $\mathfrak{A}$, and so is completely determined in terms of $\Delta$. For each $P \in P(\Delta)$ let $m(P)=\{Q \in m(\Delta): Q \subseteq P\}$ and for $P, Q \in P(\Delta)$, let $P \approx Q$ if and only if $m(P)=m(Q)$. This is clearly an equivalence relation on $P(\Delta)$. Let $S(\Delta)=P(\Delta) / \approx$; this root system is called the skeleton of $P(\Delta)$.

A branch point of a root system $\Gamma$ is an element $\eta$ of $\Gamma$ so that $\eta=\alpha \vee \beta$ for some pair of incomparable elements $\alpha, \beta$ of $\Gamma$. Therefore, $S(\Delta)$ is obtained from $P(\Delta)$ by identifying all elements of $P(\Delta)$ strictly between two adjacent branch points with the smaller branch point. Consequently, each $\sigma$ in $S(\Delta)$ is a totally ordered set. We let $P_{\sigma}=\bigcap\{Q: Q \in \sigma\}$. This is the smaller branch point and hence is the minimal element of $\sigma$. (Notice that $\bigcup\{Q: Q \in \sigma\}$ need not be an element of $\sigma$.) Thus $\sigma \rightarrow P_{\sigma}$ is a natural embedding of $S(\Delta)$ into $P(\Delta)$ which takes $P$ to $P$ for each minimal prime $P$.

Our next step in the identification of $P(\Delta)$ in terms $\Delta$ is the identification of the skeleton in those terms. To this end, we need a way to determine when a collection of minimal primes is contained in a proper prime of $V$. The following theorem gives the technique which we will use:

THEOREM 3.1. Let $\left\{P_{\varphi}: \varphi \in \Phi\right\}$ be a collection of minimal prime l-ideals of $V$. For each $\varphi$, let $\mathscr{C}_{\varphi}$ be the compatible system of ultrafilters corresponding to $P_{\varphi}$ and denote the ultrafilters on $A \in \mathfrak{A}$ belonging to $\mathscr{C}_{\varphi}$ by $A\left(\mathscr{C}_{\varphi}\right)$. Then there exists a proper prime $Q$ containing $\bigcup\left\{P_{\varphi}: \varphi \in \Phi\right\}$ if and only if there exists $A \in \mathfrak{U}$ so that $A\left(\mathscr{C}_{\varphi}\right)=A\left(\mathscr{C}_{\eta}\right)$ for all $\varphi, \eta \in \Phi$.

Proof. First, suppose that $Q \supseteq \bigcup\left\{P_{\varphi}: \varphi \in \Phi\right\}$. Choose $x \in V^{+} \backslash Q$ and $A \in \mathfrak{A}$ so that $M(x) \subseteq A$. We claim that $A\left(\mathscr{C}_{\varphi}\right)=A\left(\mathscr{C}_{\eta}\right)$ for all $\varphi, \eta \in \mathfrak{A}$. Suppose by way of contradiction that there exist $\varphi, \eta \in \Phi$ so that $A\left(\mathscr{C}_{\varphi}\right) \neq A\left(\mathscr{C}_{\eta}\right)$. Then there exists 
$X \subset A$ so that $X \in A\left(\mathscr{C}_{\varphi}\right)$, while $A \backslash X \in A\left(\mathscr{C}_{\eta}\right)$. Define $u, v \in V$ as follows:

$$
\begin{aligned}
& u(\gamma)= \begin{cases}0, & \gamma \in(\Delta \backslash A) \cup X \\
2 x(\gamma), & \gamma \in M(x) \backslash X \\
1, & \gamma \in A \backslash(X \cup M(x)),\end{cases} \\
& v(\gamma)= \begin{cases}0, & \gamma \in \Delta \backslash X, \\
2 x(\gamma), & \gamma \in M(x) \cap X, \\
1, & \gamma \in X \backslash M(x) .\end{cases}
\end{aligned}
$$

Since $M(u)=X \subseteq A, A \backslash M(u)=A \backslash X \in A\left(\mathscr{C}_{\varphi}\right)$ and so $u \in P_{\varphi}$. Similarly, $v \in P_{\eta}$. But then $u, v \in Q$ and so $u \vee v \in Q$. But $u \vee v \geqslant x$, which is not an element of $Q$, which is a contradiction. Thus, $A\left(\mathscr{C}_{\varphi}\right)=A\left(\mathscr{C}_{\eta}\right)$ for all $\varphi, \eta \in \Phi$. Let

Conversely, suppose that there exists $A \in \mathfrak{A}$ with $A\left(\mathscr{C}_{\varphi}\right)=A\left(\mathscr{C}_{\eta}\right)$ for all $\varphi, \eta \in \Phi$.

$$
S=\{v \in V: \gamma \in M(v) \text { implies that there exists } \delta \in A \text { with } \delta>\gamma\} .
$$

Then $S$ is a convex $l$-subgroup of $V$. Let $\varphi \in \Phi$ and let $Q$ be the convex $l$-subgroup of $V$ generated by $S$ and $P_{\varphi}$. Since $Q \supset P_{\varphi}, Q$ is prime. Let $\eta \in \Phi$ with $\eta \neq \varphi$, and choose $x \in P_{\eta}^{+}$. Pick $B \in \mathfrak{A}$ so that $M(x) \subseteq B$. Since $\mathscr{C}_{\varphi}$ and $\mathscr{C}_{\eta}$ are compatible systems of ultrafilters and $A\left(\mathscr{C}_{\varphi}\right)=A\left(\mathscr{C}_{\eta}\right)$, then $(A \vee B)\left(\mathscr{C}_{\varphi}\right)=(A \vee B)\left(\mathscr{C}_{\eta}\right)$. Define $v \in V$ as follows:

$$
v(\gamma)= \begin{cases}x(\gamma) & \text { if there exists } \delta \in(A \vee B) \cap M(x) \text { with } \delta \geqslant \gamma, \\ 0 & \text { otherwise. }\end{cases}
$$

Now

$$
M(v) \subseteq(A \vee B) \cap M(x) \text { and }(A \vee B) \backslash M(v)=(A \vee B) \backslash M(x) .
$$

Since $(A \vee B) \backslash M(x) \in(A \vee B)\left(\mathscr{C}_{\varphi}\right), v \in P_{\varphi}$. Clearly, $x-v \in S$. Thus $x \in Q$ and so $P_{\eta} \subseteq Q$. Since $\chi(A) \notin Q, Q$ is a proper prime of $V$ which contains $\bigcup\left\{P_{\varphi}: \varphi \in \Phi\right\}$.

For $A \in \mathfrak{A}, \mathscr{C}_{1}, \mathscr{C}_{2} \in \bar{m}(\Delta)$, we define $\mathscr{C}_{1} \sim_{A} \mathscr{C}_{2}$ if $A\left(\mathscr{C}_{1}\right)=A\left(\mathscr{C}_{2}\right)$. This is an equivalence relation on $\bar{m}(\Delta)$. Notice that if $B \geqslant A, A, B \in \mathfrak{A}$, then $\mathscr{C}_{1} \sim_{A} \mathscr{C}_{2}$ implies that $\mathscr{C}_{1} \sim_{B} \mathscr{C}_{2}$. Given $\mathscr{C} \in \bar{m}(\Delta)$, let $[\mathscr{C}]_{A}$ denote the equivalence class of $\mathscr{C}$ under $\sim_{A}$. Let

$$
\bar{S}(\Delta)=\{\sigma \subseteq \bar{m}(\Delta): \text { for all } \mathscr{C} \in \bar{m}(\Delta) \backslash \sigma,
$$

there exists $A \in \mathfrak{A}$ and $\mathscr{D} \in \bar{m}(\Delta)$ so that $[\mathscr{D}]_{A} \supseteq \sigma$ and $\left.\mathscr{C} \notin[\mathscr{D}]_{A}\right\}$.

Partially order $\tilde{S}(\Delta)$ by set inclusion. For $[P] \in S(\Delta)$, let

$$
f([P])=\left\{\mathscr{C}_{Q} \in \bar{m}(\Delta): Q \in m(P)\right\}
$$

and if $\sigma \in \bar{S}(\Delta)$, let $g(\sigma)=\left[P_{\sigma}\right]$, where $P_{\sigma}=\bigcap\left\{P \in P(\Delta): P \supseteq P_{\mathscr{G}}\right.$, for all $\left.\mathscr{C} \in \sigma\right\}$. 
THEOREM 3.2. $f$ is an order isomorphism of $S(\Delta)$ onto $\bar{S}(\Delta)$ with inverse $g$.

Proof. We first show that $f([P]) \in \bar{S}(\Delta)$. Let $\mathscr{C} \in \bar{m}(\Delta) \backslash f([P])$. Since $P_{\mathscr{C}} \neq P$, there exists $x \in P_{\mathscr{C}}^{+} \backslash P$. Choose $A \in \mathfrak{U}$ so that $M(x) \subseteq A$. By the proof of Theorem 3.1, $\left[\mathscr{C}_{Q}\right]_{A} \supseteq f([P])$, for each $Q \in m(P)$. Let $Q \in m(P)$. Since $x \notin Q$, there exists $B \in \mathfrak{U}$ so that $B \backslash M(x) \notin B\left(\mathscr{C}_{Q}\right)$. Therefore $M(x) \cap B \in B\left(\mathscr{C}_{Q}\right)$. Since $M(x) \subseteq A$ and

$$
M(x) \notin A(\mathscr{C}), \quad A(\mathscr{C}) \neq A\left(\mathscr{C}_{Q}\right) .
$$

Thus, $\mathscr{C} \notin\left[\mathscr{C}_{Q}\right]_{A}$ and so $f([P]) \in \bar{S}(\Delta)$.

Clearly $g f([P])=[P]$ and both $f$ and $g$ preserve order; it remains to show that $f g(\sigma)=\sigma$. We need only check that if $\sigma \in \bar{S}(\Delta)$, then $m\left(P_{\sigma}\right)=\left\{P_{\mathscr{C}}: \mathscr{C} \in \sigma\right\}$. One containment is clear. Suppose (by way of contradiction) that $P_{\sigma} \supseteq Q$ for some $Q \in m(\Delta)$ with $\mathscr{C}_{Q} \notin \sigma$. Then there is an $A \in \mathfrak{U}$ and $\mathscr{D} \in \bar{m}(\Delta)$ so that $[\mathscr{D}]_{A} \supseteq \sigma$ but $\mathscr{C}_{Q} \notin[\mathscr{D}]_{A}$. By the proof of Theorem 3.1, there is a prime $N \supset \bigcup\left\{P_{\mathscr{C}}: \mathscr{C} \in \sigma\right\}$ with $Q \nsubseteq N$. Therefore $N \subset P_{\sigma}$ which contradicts the definition of

$$
P_{\sigma}=\bigcap\left\{P: P \supseteq P_{\mathscr{C}}, \mathscr{C} \in \sigma\right\} .
$$

We have now seen that the skeleton $S(\Delta)$ is describable entirely in terms of $\Delta$. It now remains to describe the primes in each $[P] \in S(\Delta)$.

Let $[P] \in S(\Delta)$ and suppose $\sigma=f([P]) \subseteq \bar{m}(\Delta)$. Define

$$
B(\sigma)=\left\{A \in \mathfrak{U}: \mathscr{C}_{1} \sim_{A} \mathscr{C}_{2}, \text { for all } \mathscr{C}_{1}, \mathscr{C}_{2} \in \sigma\right\} .
$$

Notice that if $A \in B(\sigma)$ and $B \geqslant A$, then $B \in B(\sigma)$. For notational convenience, for each $B \in B(\sigma)$, let $B(\mathscr{U})=B\left(\mathscr{U}_{P_{\mathscr{C}}}\right)$ where $\mathscr{C}$ is any element of $\sigma$. (This is possible by the definition of $B(\sigma)$.) For $A, B \in B(\sigma)$, let $A^{B}=\{\alpha \in A: \alpha<\beta \in B\}$, and let $A_{B}=\{\alpha \in A: \alpha>\beta \in B\}$. (Another description of $A^{B}$ is $\left.A^{B}=((A \wedge B) \cap A) \mid(A \cap B)\right)$. Now, $A=A^{B} \cup A_{B} \cup(A \cap B)$ and precisely one of these sets is in $A(\mathscr{U})$. Define $A \sim B$ if $A \cap B \in A(\mathscr{U})(A, B \in B(\sigma))$. This is an equivalence relation on $B(\sigma)$. If $A^{B} \in A(\mathscr{U})$, then we write $[A] \prec[B]$, where the brackets denote the equivalence class under $\sim$. A routine computation shows that this relation is well defined and forms a total order on $B(\sigma) / \sim$.

LEMMA 3.3. If $A, B \in B(\sigma)$ with $[A] \geqslant[B]$, then $A \vee B \sim A$. Therefore, if $[A] \geqslant[B]$, we may assume that $A \geqslant B$.

Proof. Suppose that $[A] \geqslant[B]$. Now, $(A \vee B) \cap A \supseteq A_{B} \cup(A \cap B)$. If $[A] \succ[B]$, then $A_{B} \in A(\mathscr{U})$; if $[A]=[B]$, then $A \cap B \in A(\mathscr{U})$. In either case, $(A \vee B) \cap A \in A(\mathscr{U})$ and so $A \vee B \sim A$.

For each $A \in B(\sigma)$, let

$$
P_{A}=\{v \in V: \text { for all } B \geqslant A, B \backslash M(v) \in B(\mathscr{U})\} .
$$


Lemma 3.4. $P_{A}$ is a convex l-subgroup of $V$ containing $P_{\sigma}$.

Proof. Let $S=\{v \in V: \gamma \in M(v) \Rightarrow$ there exists $\delta \in A$ with $\delta>\gamma\}$ and let $\mathscr{C} \in \sigma$. A routine argument shows that $P_{A}$ is the convex $l$-subgroup generated by $S$ and $P_{\mathscr{C}}$, and the proof of Theorem 3.1 shows that $P_{A} \supseteq \bigcup\left\{P_{\mathscr{C}}: \mathscr{C} \in \sigma\right\}$. Since $P_{\sigma}$ is the intersection of all such $P_{\mathscr{C}}, P_{A} \supseteq P_{\sigma}$.

Proposition 3.5. Let $A, B \in B(\sigma)$. Then

(i) $P_{B}=P_{A}$ if and only if $[B]=[A]$.

(ii) $P_{B} \subset P_{A}$ if and only if $[B] \prec[A]$.

Proof. We will first show that if $[A]=[B]$ and $B \geqslant A$, then $P_{A}=P_{B}$. By the definition of $P_{A}, P_{A} \subseteq P_{B}$. Let $D \geqslant A$ and let $x \in V$ be chosen so that

$$
D \cap M(x) \in D(\mathscr{U})
$$

(that is, $\left.x \notin P_{A}\right)$. Since $[A]=[B], A \cap B \in A(\mathscr{U})$ and since $D \geqslant A, D^{+}(A \cap B) \in D(\mathscr{U})$. But then $D^{\leftarrow}(A \cap B) \cap M(x) \in D(\mathscr{U})$. Since

$$
D^{\leftarrow}(A \cap B) \subseteq B \vee D, \quad D^{\leftarrow}(A \cap B) \cap M(x) \in(B \vee D)(\mathscr{U}) .
$$

Therefore $x \notin P_{B}$, and so $P_{A}=P_{B}$.

If $[B] \geqslant[A]$, we may assume that $B \geqslant A$, by Lemma 3.3 and the above. Then $P_{B} \supseteq P_{A}$, by definition. This shows that if $P_{B} \subset P_{A}$, then $[B] \prec[A]$. Now, suppose that $[B] \prec[A]$. We may assume that $B<A$, and so $A=A_{B} \cup(A \cap B)$. Since $[B] \prec[A], \quad B^{A} \in B(\mathscr{U})$. Therefore, $\chi\left(B^{A}\right) \notin P_{B}$. Since $C \backslash M\left(\chi\left(B^{A}\right)\right)=C$ for all $C \geqslant A, \chi\left(B^{A}\right) \in P_{A} \backslash P_{B}$. Because $P_{A}$ and $P_{B}$ are comparable, $P_{A} \supset P_{B}$.

Finally, if $[A] \neq[B]$, then without loss of generality $[A] \prec[B]$. Consequently, by part (ii), $P_{A} \neq P_{B}$ and so $P_{A}=P_{B}$ implies that $[A]=[B]$.

Proposition 3.6. $P_{\sigma}=\bigcap\left\{P_{A}: A \in B(\sigma)\right\}$.

Proof. Let $0<v \in \bigcap\left\{P_{A}: A \in B(\sigma)\right\}$. If $M(v) \subseteq A \in B(\sigma)$, then $v \in P$ for all $\mathscr{C} \in \sigma$. Thus $v \in P_{\sigma}$. If $M(v) \subseteq A \notin B(\sigma)$, then there exist $\mathscr{C}_{1}, \mathscr{C}_{2} \in \sigma$ such that $\mathscr{C}_{1} \sim_{A} \mathscr{C}_{2}$. Thus, there exists $X \subset A$ so that $X \in A\left(\mathscr{C}_{1}\right)$ and $A \backslash X \in A\left(\mathscr{C}_{2}\right)$. Define $w_{1}, w_{2} \in V$ as follows:

$$
\begin{aligned}
& w_{1}(\gamma)= \begin{cases}v(\gamma) & \text { if } \gamma \in X, \\
0 & \text { if } \gamma \in \Delta \backslash X,\end{cases} \\
& w_{2}(\gamma)= \begin{cases}\nu(\gamma) & \text { if } \gamma \in A \backslash X, \\
0 & \text { if } \gamma \in \Delta \backslash(A \backslash X) .\end{cases}
\end{aligned}
$$

Then $A \backslash M\left(w_{1}\right) \supseteq A \backslash X \in A\left(\mathscr{C}_{2}\right)$ and so $w_{1} \in P_{\mathscr{C}_{2}}$; similarly $w_{2} \in P_{\mathscr{C}_{1}}$. Therefore 
$w_{1}+w_{2} \in P_{\sigma}$. Since $w_{1}+w_{2} \geqslant v>0, v \in P_{\sigma}$. Thus $P_{\sigma} \supseteq \bigcap\left\{P_{A}: A \in B(\sigma)\right\}$. The other containment follows from Lemma 3.4.

Proposition 3.7. Suppose $A, B \in B(\sigma)$ and $A \sim B$. Let

and

$$
Q_{A}=\left\{f \in \Pi_{A} \mathbf{R}: A \backslash S(f) \in A(\mathscr{U})\right\}
$$

$$
Q_{B}=\left\{f \in \Pi_{B} \mathbf{R}: B \backslash S(f) \in B(\mathscr{U})\right\} .
$$

(These are minimal primes of $\Pi_{A} \mathbf{R}$ and $\Pi_{B} \mathbf{R}$ respectively.) Then $\Pi_{A} \mathbf{R} / Q_{A}$ and $\Pi_{B} \mathbf{R} / Q_{B}$ are isomorphic o-groups.

Proof. We define $\mu: \Pi_{A} \mathbf{R} / Q_{A} \rightarrow \Pi_{B} \mathbf{R} / Q_{B}$ as follows: Given $Q_{A}+v \in \Pi_{A} \mathbf{R} / Q_{A}$, define $w \in \Pi_{B} \mathbf{R}$ by

$$
w(\beta)= \begin{cases}v(\alpha) & \text { if } \beta \leqslant \alpha \in A, \beta \in B^{A} \cup(A \cap B), \\ 0 & \text { otherwise. }\end{cases}
$$

Then let $\mu\left(Q_{A}+v\right)=Q_{B}+w$.

First we show that $\mu$ is well defined: If $v \in Q_{A}$, then $A \backslash S(v) \in A(\mathscr{U})$. Now,

$$
\begin{aligned}
B \backslash S(w) & =B \backslash\{\beta \in B: \beta \leqslant \alpha \in S(v)\} \\
& =\{\beta \in B: \beta>\alpha \in A\} \cup\{\beta \in B: \beta \leqslant \alpha \in A \backslash S(v)\} \\
& \supseteq\{\beta \in B: \beta>\alpha \in A \backslash S(v)\} \cup\{\beta \in B: \beta \leqslant \alpha \in A \backslash S(v)\} .
\end{aligned}
$$

Since $A(\mathscr{U})$ and $B(\mathscr{U})$ are compatible ultrafilters and $A \backslash S(v) \in A(\mathscr{U})$,

$$
\{\beta \in B: \beta>\alpha \in A \backslash S(v)\} \cup\{\beta \in B: \beta \leqslant \alpha \in A \backslash S(v)\} \in B(\mathscr{U}) .
$$

Hence $w \in Q_{B}$ and so $\mu$ is well defined.

We define $\nu: \Pi_{B} \mathbf{R} / Q_{B} \rightarrow \Pi_{A} \mathbf{R} / Q_{A}$ similarly, and claim that $\nu \mu\left(Q_{A}+v\right)=Q_{A}+v$. By definition, $\nu \mu\left(Q_{A}+v\right)=Q_{A}+\left.v\right|_{A \cap B}$ where $\left.v\right|_{A \cap B}(\gamma)=v(\gamma)$ if $\gamma \in A \cap B$ and is 0 if $\gamma \notin A \cap B$. Therefore, we need to show that $A \backslash S\left(v-\left.v\right|_{A \cap B}\right) \in A(\overline{\mathscr{U}})$. Since $S\left(v-\left.v\right|_{A \cap B}\right) \subseteq A \backslash(A \cap B)$,

$$
A \backslash S\left(v-\left.v\right|_{A \cap B}\right) \supseteq A \backslash(A \backslash A \cap B)=A \cap B \in A(\mathscr{U}) .
$$

Thus $\nu \mu\left(Q_{A}+v\right)=Q_{A}+v$. Similarly, $\mu \nu$ is the identity on $\Pi_{B} \mathbf{R} / Q_{B}$. Since $\mu$ clearly preserves order, $\mu$ is an $o$-isomorphism.

This proposition enables us to define an $o$-group which we will use to analyze the order structure of $[P] \in S(\Delta)$. Let $\sigma=f([P]) \in \bar{S}(\Delta)$. Then set $G_{[A]}=\Pi_{A} \mathbf{R} / Q_{A}$, for each $[A] \in B(\sigma) / \sim$. This is well defined by Proposition 3.7. Let

$$
H_{\sigma}=V\left(B(\sigma) / \sim, G_{[A]}\right)=\left\{k \in \Pi\left\{G_{[A]}: A \in B(\sigma) / \sim\right\}: S(k) \text { satisfies the } A C C\right\},
$$


where $S(k)$ is given the total order $\prec$ inherited from $B(\sigma) / \sim$, and $H$ has the obvious $o$-group structure.

THEOREM 3.8. There exists an o-monomorphism

$$
\iota: V / P_{\sigma} \rightarrow H_{\sigma}
$$

so that $H_{\sigma}$ is an a-extension of $\iota\left(V / P_{\sigma}\right)$.

Proof. We define $\iota\left(P_{\sigma}+v\right)([A])=Q_{A}+\left.v\right|_{A}$.

We first show $\iota$ is well defined into $\Pi G_{[A]}$. Suppose $v \in P_{\sigma}$ and $A \in B(\sigma)$. Choose $B \geqslant A$ so that

$$
X=\left\{\beta \in M(v): \beta \geqslant \alpha \in M\left(\left.v\right|_{A}\right)\right\} \subseteq B .
$$

If $M\left(\left.v\right|_{A}\right) \in A(\mathscr{U})$, then $X \in B(\mathscr{U})$ since the $A(\mathscr{U})$ and $B(\mathscr{U})$ are compatible and $B^{\leftarrow}\left(M\left(\left.v\right|_{A}\right)\right)=X$. However, $X \cap(B \backslash M(v))=\varnothing$ and since $v \in P_{\sigma}, B \backslash M(v) \in B(\mathscr{U})$. Consequently, $X \notin B(\mathscr{U})$ and so $M\left(\left.v\right|_{A}\right) \notin A(\mathscr{U})$. Therefore, $\left.v\right|_{A} \in Q_{A}$ and $\iota$ is well defined into $\Pi G_{[A]}$.

We claim that $\iota$ is one-to-one into $H$. Suppose $P_{\sigma}+v>0$ and choose $B \supseteq M(v)$. Since $v \notin P_{\sigma}$, there exists $A \in B(\sigma)$ so that $M(v) \cap A \in A(\mathscr{U})$. Clearly $A \cap M(v) \subset B \vee A$ and since $A(\mathscr{U})$ and $(B \vee A)(\mathscr{U})$ are compatible, $M(v) \cap A \in A(\mathscr{U})$ implies that $A \cap M(v) \in(A \vee B)(\mathscr{U})$. Therefore,

$$
(A \vee B) \backslash M\left(\left.v\right|_{A \vee B}\right) \notin(A \vee B)(\mathscr{U}),
$$

and so $\left.v\right|_{A \vee B} \notin Q_{A \vee B}$. Therefore $\iota\left(P_{\sigma}+v\right)([A \vee B])$ is not zero and so $\iota$ is one-to-one. We now claim that $[A \vee B]$ is the maximum element of $S\left(\iota\left(P_{\sigma}+v\right)\right)$ and so $\iota\left(P_{\sigma}+v\right) \in H$. Suppose $[C] \succ[A \vee B]$ where (without loss of generality) $C>A \vee B$. Since $M(v) \subseteq B, S\left(\left.v\right|_{C}\right) \subseteq C \cap(A \vee B)$. Because $[C] \succ[A \vee B]$,

$$
X=\{\gamma \in C: \gamma>\alpha \in A \vee B\} \in C(\mathscr{U}),
$$

and because $C \backslash S\left(\left.v\right|_{C}\right) \supset X, C \backslash S\left(\left.v\right|_{C}\right) \in C(\mathscr{U})$. Therefore $\left.v\right|_{C} \in Q_{C}$ and so

$$
\iota\left(P_{\sigma}+v\right)([C])=Q_{C}+0 .
$$

Thus $[C] \notin S\left(\iota\left(P_{\sigma}+v\right)\right)$.

Finally, since $\iota\left(V / P_{\sigma}\right) \supseteq \Sigma G_{[A]}, H_{\sigma}$ is an $a$-extension of $\iota\left(V / P_{\sigma}\right)$.

REMARK. The map $\iota$ is independent of which representative of $[A]$ we choose to define the component maps, because of the nature of the isomorphisms

$$
\Pi_{A} \mathbf{R} / Q_{A} \rightarrow \Pi_{B} \mathbf{R} / Q_{B} .
$$

Now suppose that $\sigma \in \bar{S}(\Delta)$. Let

$$
A(\sigma)=B(\sigma) \backslash \bigcup\{B(\tau): \tau \in \bar{S}(\Delta) \text { and } \tau \supset \sigma\}
$$


and let

$$
M_{\sigma}=\bigcap\left\{P_{A}: A \in B(\sigma) \backslash A(\sigma)\right\}=\bigcap\left\{P_{\tau}: \tau \in \bar{S}(\Delta), \tau \supset \sigma\right\} .
$$

If there exists a smallest $\tau$ so that $\tau \supset \sigma$, then $M_{\sigma}=P_{\tau}$ and $M_{\sigma} \notin\left[P_{\sigma}\right]$. If no such $\tau$ exists, then $M_{\sigma}$ is the largest element of the chain $\left[P_{\sigma}\right]$. In particular, if $A(\sigma)=\varnothing$, then $M_{\sigma}=P_{\sigma}$ and so $\left[P_{\sigma}\right]$ is a singleton.

Thus, the elements of $\left[P_{\sigma}\right]$ are in a one-to-one correspondence with $\mathscr{C}\left(M_{\sigma} / P_{\sigma}\right)$, the convex subgroups of $M_{\sigma} / P_{\sigma}$, except possibly for the existence of a largest element as specified above. However, $M_{\sigma} / P_{\sigma}$ is the convex subgroup of $V / P_{\sigma}$ which corresponds to $V\left(A(\sigma) / \sim, G_{[A]}\right)$ under the $a$-extension of Theorem 3.8 .

Thus, we now need a way of describing the convex subgroup structure of the Hahn group $V\left(\Gamma, G_{\gamma}\right)$ where $\Gamma$ is a totally ordered set and each $G_{\gamma}$ is an $o$-group with $\mathscr{C}\left(G_{\gamma}\right)$ its set of convex subgroups. Let

$$
\mathscr{G}=\bigcup\left\{\{\gamma\} \times \mathscr{C}\left(G_{\gamma}\right): \gamma \in \Gamma\right\}
$$

If $\alpha, \beta \in \Gamma$ with $\alpha$ covering $\beta$, we will identify $(\alpha, 0)$ with $\left(\beta, G_{\beta}\right)$. Call $\mathscr{G}$ modulo this equivalence relation $\mathscr{H}$ and order it lexicographically with the first component dominating. Clearly $\mathscr{C}\left(V\left(\Gamma, G_{\gamma}\right)\right)$ is order isomorphic to $\mathscr{H}$.

Thus, we have described $P(\Delta)$ up to the convex subgroup structure of the $o$-groups $G_{[A]}$. But $G_{[A]}=\Pi_{A} \mathbf{R} / Q_{A}$. Now, $Q_{A}$ is a maximal ring ideal of $\Pi_{A} \mathbf{R}$, considered as the ring of continuous functions on the discrete space $A$ (see Bigard, Keimel and Wolfenstein (1977), p. 179), and so $G_{[A]}$ is a real-closed $\eta_{1}$-field (see Gillman and Jerison (1960)). Now, we claim that $\Gamma\left(G_{[A]}\right)$ (the values of $G_{[A]}$ ) is an $\eta_{1}$-set. For, if

$$
P_{1} \subset P_{2} \subset P_{3} \ldots Q_{3} \subset Q_{2} \subset Q_{1}
$$

are all values, choose $g_{i}, h_{j} \in G_{[A]}^{+}$such that $P_{i}$ is the value of $g_{i}$ and $Q_{j}$ is the value of $h_{j}$. Then $\left\{g_{i}\right\}<\left\{h_{j}\right\}$ and so there exists $k \in G_{[A]}^{+}$with $\left\{g_{i}\right\}<k<\left\{h_{j}\right\}$. Thus, the value of $k$ lies between the $P_{i}$ 's and $Q_{j}$ 's. But $\mathscr{C}\left(G_{[A]}\right)$ is just the Dedekind-MacNeille completion of $\Gamma\left(G_{[A]}\right)$, considered as a totally ordered set. Consequently, we have concluded that $\mathscr{C}\left(G_{[A]}\right)$ is in each case the Dedekind-MacNeille completion of an $\eta_{1}$-set.

Nore. Portions of this paper first appeared in the second author's Ph.D. dissertation 'Lattice-ordered groups', written at the University of Kansas in 1976 under the direction of Dr. Paul F. Conrad.

\section{References}

A. Bigard, K. Keimel and S. Wolfenstein (1977), Groupes et anneaux réticules (Springer-Verlag, Berlin).

P. Conrad (1970), Lattice-ordered groups (Tulane Library, New Orleans). 
P. Conrad (1978), 'Minimal prime subgroups of lattice-ordered groups', preprint.

P. Conrad, J. Harvey and C. Holland (1963), 'The Hahn embedding theorem for latticeordered groups', Trans. Amer. Math. Soc. 108, 143-169.

P. Conrad and D. McAlister (1969), 'The completion of a lattice-ordered group', J. Austral. Math. Soc. 9, 182-208.

L. Gillman and M. Jerison (1960), Rings of continuous functions (van Nostrand, Princeton).

Department of Mathematical Sciences

Indiana University-Purdue University at Fort Wayne

2101 Coliseum Boulevard East

Fort Wayne, Indiana 46805

U.S.A.
Department of Mathematics Boise State University Boise, Idaho 83725 U.S.A. 